\title{
ESTUDIO DE DIGESTIBILIDAD APARENTE DE LA HARINA DE LOMBRIZ (Eisenia andrei) EN LA ALIMENTACIÓN DE TRUCHA ARCO IRIS (Onchorinchus mykiss)
}

\section{APPARENT DIGESTIBILITY STUDY OF THE FLOUR OF EARTHWORM (Eisenia andrei) IN THE FEEDING OF RAINBOW TROUT (Onchorinchus mykiss)}

Fernando Isea L (1), Célestin Blé M (3), Ana Luisa Medina G (2), Pierre Aguirre (4), Guillermo Bianchi P (2) Sadasivam Kaushik (4)

(1) Programa Doctoral en Ciencias Aplicadas. Facultad de Ingeniería Universidad de los Andes, Venezuela.

(2) Grupo Ecología y Nutrición. Departamento de Ciencia de los Alimentos. Facultad de Farmacia y Bioanálisis. ULA-Mérida. Venezuela.

(3) Centre de Recherches Océanologique (CRO). Departament de aquaculture. Cotê d'Ivoire.

(4) Institut National de la Recherche Agronomique (INRA) [Instituto Nacional de Investigaciones Agronomicas], Saint Pée sur Nivelle. Francia.

Este trabajo fue recibido el 31 de Julio de 2007 y aceptado para ser publicado el 16 de Enero de 2008.

Dirigir la correspondencia a:

Profesor

Fernando Isea L.

Facultad de Ingeniería

Universidad de los Andes

ULA-Mérida 5101-Venezuela

Fono Fax: 58-274-2403473.

E-mail: fernandoisea@yahoo.es 


\begin{abstract}
The primary objective of this investigation was to determine the apparent digestibility coefficient (ADC) of earthworm flour (EW) and compare it with other raw feeding materials already evaluated like wheat bran (BW) and soy cake (CS) in the feeding of the rainbow trout (Oncorhynchus mykiss). Fifteen trout of an average weight of $100 \mathrm{~g}$ were placed in duplicate tanks provided with an automated fecal material collecting system. CS and EW flour showed a protein $\mathrm{ADC}$ of $90.1 \%$ and $90.0 \%$, respectively, when comparing them with BW (57.2\%). Energy ADC was superior in CS $(75.4 \mathrm{KJ} / \mathrm{g})$ and $\mathrm{EW}(72.5 \mathrm{KJ} / \mathrm{g})$. The values of he percentage of digestible protein were superior in the EW (63.4\%); in comparison with CS (48.3\%) and AT (8.8\%); digestible energy was superior in EW $(17.1 \mathrm{KJ} / \mathrm{g})$, followed by CS $(14.9 \mathrm{KJ} / \mathrm{g})$ and AT $(2.4 \mathrm{KJ} / \mathrm{g})$. We conclude that the EW flour has an excellent protein and energy digestibility.
\end{abstract}

Key words: Oncorhynchus mykiss; digestibility; earthworm; soybean

\title{
INTRODUCCION
}

El alimento para peces representa hasta un $40 \%$ de los costos operacionales de las pisciculturas comerciales, en general, la expansión en la acuacultura ha incrementado los precios drásticamente en los últimos años, por la escasez de recursos marinos (1). El mayor costo lo refleja la harina de pescado, materia prima de referencia, por lo cual han surgido numerosas investigaciones con la finalidad de evaluar alternativas viables de sustitución de la misma, en la manufactura de dietas para peces (2-4). La proteína para los peces es uno de los componentes energéticos más importantes a considerar y por consiguiente se debe tomar en cuenta las fuentes posibles de esta a ser usadas en las dietas (5). Vanderberg y De la Noüe, (6), refieren que se necesita información relativa a la disponibilidad de nutrientes específicos para llevar a cabo estudios de requerimientos y evaluación de insumos como posibles candidatos de inclusión en dietas que tengan como 
característica su bajo costo o costo moderado de fabricación y generen un mínimo impacto en la contaminación del medio ambiente.

La necesidad de herramientas confiables para estudiar la utilización de ingredientes lleva al desarrollo de varios métodos para entender el grado en que los nutrientes son absorbidos, incluidas las mediciones de digestibilidad aparente de los nutrientes. La digestibilidad de una materia prima o de un alimento representa la cantidad absorbida reportada a la cantidad ingerida, y puede ser estimada por diferencia en la fracción eliminada en las heces (7).

Entre las especies de peces, la trucha arco iris ha sido una de las mas usadas en estudios de determinación de digestibilidad aparente de materias primas (CUDa), tanto de origen animal como vegetal. Hilton (5) obtuvo un coeficiente de digestibilidad aparente de 69,2 \% y su CUDa proteica fue de $95 \%$ mediante el uso de harina de lombriz (Eudrilus eugenige). Por su parte Tacon y cols (8), obtuvo incrementos progresivos de la digestibilidad del nitrógeno (proteína) mediante el uso de harina de lombriz (Eisenia foetida) al ser comparada con el control comercial, por lo cual señalan que esta es una buena alternativa como fuente de proteína animal para formulaciones alimenticias de peces. Resultados similares fueron obtenidos por Stafford y Tacon (9), con dietas para truchas fortificadas $(5,10,20$ y $30 \%)$ con harina de lombriz con resultados de CUDa de 94,31 a 95,26\% que superaron al control $(93,05)$, a medida que se incrementaba la dosis de un $5 \%$ a un $30 \%$. Por lo antes mencionado, se efectuó un estudio con el objeto de evaluar la digestibilidad aparente de la harina de lombriz (Eisenia andrei) comparada con otras dos materias primas vegetales (torta de soya y afrecho de trigo) en trucha arco iris.

\section{MATERIALES Y MÉTODO}

El presente trabajo se efectuó en la sala experimental de estudio de digestibilidad de la estación del INRA, Saint Pee sur Nivelle, Quartier Ibarron, Francia. 


\section{Preparación de alimentos}

Se sometieron a evaluación cuatro (4) alimentos, tres (3) de ellos con diferentes materias primas (harina de lombriz, harina de afrecho de trigo y harina de torta de soja) sustituyendo un $30 \%$ de la composición del alimento de referencia (alimento preparado a base de harina de pescado Noruego). Para ello se tomó un $70 \%$ del alimento base con previa adición del marcador óxido de cromo III $\left(\mathrm{Cr}_{2} \mathrm{O}_{3}\right)$ al $1,5 \%$ (para efectos de medir la digestibilidad aparente), al cual se le complementó en un $30 \%$ de cada materia prima. En la tabla 1 se muestra la formulación del alimento de referencia.

\section{TABLA 1}

Formulación (g/100g) del alimento de referencia

\begin{tabular}{lc}
\hline \multicolumn{1}{c}{ Ingrediente } & Alimento Referencia \\
\hline Harina de pescado noruego & 60 \\
Aceite de pescado & 7 \\
Almidón gelatinizado & 27,5 \\
Mezcla de vitaminas & 1 \\
Mezcla de minerales & 1 \\
Ligante (Alginato de sodio) & 2 \\
Oxido de cromo & 1,5 \\
Harina de lombriz & 0 \\
Torta de soja & 0 \\
Afrecho de trigo & 0 \\
\hline Total & 100 \\
\hline
\end{tabular}


Luego de definir la cantidad de cada componente, se procedió a la preparación de las dietas en la sala de fabricación de alimentos del INRA Saint Pee sur Nivelle, donde se mezclaron los ingredientes y se fabricaron los gránulos que posteriormente fueron secados y conservados bajo refrigeración $\left(4^{\circ} \mathrm{C}\right)$ hasta su utilización posterior. De estos granulados se tomó una muestra representativa para realizar el análisis proximal y de fósforo que permitió conocer los valores prácticos de la composición química de los mismos, y la determinación del contenido de óxido de cromo, traceador indigestible necesario para determinar indirectamente la digestibilidad aparente de cada componente en las dietas evaluadas.

El análisis proximal (porcentajes de humedad, ceniza, proteína, grasa) y la determinación de fósforo y oxido de cromo, se obtuvo siguiendo el protocolo establecido en el Manual de Laboratorio del INRA, Saint Pée sur Nivelle. Los equipos usados fueron los siguientes: Estufa Memmert, calibrada a $110{ }^{\circ} \mathrm{C}$; Equipo de vacio para desecador KNF Neuberger, modelo LABOPORT; Kjeltec Analyzer Unit, modelo 2300, Foss Tecator; Digestor FOSS, modelo 2040, Based Tecator ${ }^{\mathrm{TM}}$ Technology; Zoster automatic, marca Gerhardt; Baño de Maria Gallenkamp Regol; Sauerstoff Füllstation C48; Calorimeter IKA, modelo C 4000, adiabatic, IKA ${ }^{\circledR}$ Analysentechnik Heitersheim; Termo Haake K10; Termo Haake DC 10; Balanza EM-3000 (3 kg x 1 g), Matfer; Balance Analitical, Sartorius BP2215 max. 220 g d= 0.1 mg y la Mufla: LENTON Turnase, thermal designs $\operatorname{LTD}\left(550^{\circ} \mathrm{C}\right)$. El óxido de cromo se determinó mediante un espectrofotómetro UV-visible, 160 A, SHIMADZU, a $440 \mathrm{~nm}$. La concentración del fósforo se determinó por medio de un espectrofotómetro UV-Visible, marca SHIMADZU, modelo UV1205. Ambos componentes mediante el protocolo del manual del INRA. 


\section{Condiciones experimentales}

Se utilizaron ejemplares de trucha arco iris (Oncorhynchus mykiss) de un peso promedio de 100 $\mathrm{g}$, en total quince (15) truchas por tanque. Los tratamientos se evaluaron por duplicado. En este trabajo se emplearon ocho (8) tanques cilíndricos de fibra de poliéster, sus diámetros fueron de 40 cm de diámetro y de base cónica que facilita la sedimentación de las heces. Estos tanques estuvieron ubicados en la sala experimental de estudio de digestibilidad, con una capacidad volumétrica de $60 \mathrm{~L}$, alimentados con caudales de agua filtrada y termoregulada de $4 \mathrm{~L} / \mathrm{min}$, a una temperatura de $17 \pm 1^{\circ} \mathrm{C}$. La colección automática de heces en los tanques permitió la obtención de muestras en condiciones óptimas, debido a las dificultades de colectar materiales sólidos en agua.

\section{Alimentación y recolección de heces}

Los peces fueron sometidos a una adaptación previa de una semana (20/03/06-26-/03/06), con el objeto de vaciar completamente el sistema digestivo y al mismo tiempo lograr la adaptación de estos al nuevo sistema de cría cilíndrico, favoreciendo la toma de alimentos durante el ensayo. La alimentación fue manual "ad-libitum", efectuada dos veces por día (9 am y 4 pm) los siete días de la semana, con una duración de 2 semanas. Durante la alimentación se cuidó de que todo el alimento suministrado fuera consumido, lo que se efectuó observando los recipientes de recolecta (coladores plásticos) donde se devolvían los gránulos, que no fueron consumidos, posterior al suministro, para evitar una posible contaminación de las heces con el alimento no consumido. Las heces fueron colectadas en recipientes de aluminio cada día en la mañana y en la tarde, congeladas y liofilizadas para posteriormente realizar análisis respectivos. 


\section{Evaluación del Coeficiente de Digestibilidad aparente (CUDa)}

- Para el cálculo de CUDa (\% materia seca), CUDa de nutrientes (\%) y CDA energía (KJ/g) de los alimentos, se emplearon las ecuaciones extraídas del trabajo de Maynard y Loosli (10):

$$
\begin{gathered}
\text { CUDa MS }(\%)=100\left[1-\left(\frac{\% \mathrm{Cr}_{2} \mathrm{O}_{3} \text { del al imento }}{\% \mathrm{Cr} \mathrm{O}_{3} \text { de las heces }}\right)\right] \\
\text { CUDa nutrientes }=100\left[1-\left(\frac{\% \mathrm{Cr}_{2} \mathrm{O}_{3} \text { del al imento }}{\% \mathrm{Cr}_{2} \mathrm{O}_{3} \text { de las heces }}\right) *\left(\frac{\% \text { nutrientes de heces }}{\% \text { nutrientes del al imento }}\right)\right]
\end{gathered}
$$

- Para el cálculo de CUDa (Materia seca, nutrientes y energía) de las materias primas (MP) se emplearon las ecuaciones extraídas del trabajo de Sugiura et al., (11) basadas sobre la teoría que las digestibilidades son aditivas.

\begin{tabular}{|c|c|c|c|c|c|}
\hline CUDa de nutriente & {$\left[\begin{array}{l}\text { Concetracin de nuriente } \\
\text { oenergia al imento teste }\end{array}\right] *$} & {$\left[\begin{array}{c}\text { CUDa nutrienteonergia } \\
\text { alimentotestado }\end{array}\right]$} & $-0,7 *$ & {$\left[\begin{array}{l}\text { Nutrienteo energia } \\
\text { alimento de referencia }\end{array}\right]$} & {$\left[\begin{array}{c}\text { CUDa denutrienteo energia } \\
\text { dealimentode referencia }\end{array}\right]$} \\
\hline yenergia & \multicolumn{5}{|c|}{ lenuriente } \\
\hline
\end{tabular}

CUDa de materia seca de $M P(\%)=\frac{C U D a \text { Al imento teste }-0,7 * \text { CUDa Alimento referencia }}{0,3}$

\section{Análisis estadístico}

Cada muestra se analizó por triplicado, y se realizó un análisis varianza, con un nivel de significancia de $\mathrm{p}<0,001$. También se aplicó la Prueba de Rango Múltiple de Duncan con $\alpha=$ 0,05, para la determinación de subconjuntos homogéneos o no. Se compararon los coeficientes de digestibilidad de la harina de lombriz y de la torta de soja mediante una prueba t para muestras independientes con varianzas desiguales. Estos cálculos se efectuaron utilizando el programa estadístico SPSS versión 10, para Windows. 


\section{RESULTADOS Y DISCUSIÓN}

La tabla 2 muestra la composición química de los alimentos en estudio. El análisis de varianza detectó diferencias significativas $(\mathrm{p}<0,001)$ en la composición proximal de las dietas evaluadas. La prueba de rango múltiple de Duncan detectó diferencias significativas $(p<0,05)$ entre algunas dietas. El nivel proteico del alimento con afrecho de trigo es significativamente inferior a los tres alimentos AR, T1 y T2. En consecuencia similares resultados se obtuvieron para el parámetro de energía y materia seca.

\section{TABLA 2}

Composición química de las dietas evaluadas $(\bar{x} \pm D E$. $)$

\begin{tabular}{|c|c|c|c|c|}
\hline Determinación & $\overline{\mathbf{A R}}$ & T1 & $\mathbf{T 2}$ & T3 \\
\hline$\%$ Materia seca $(\mathrm{MS})$ & $90,5^{b} \pm 0,10$ & $94,0^{b} \pm 0,01$ & $91,3^{b} \pm 0,01$ & $89,4^{\mathrm{a}} \pm 0,18$ \\
\hline Energía (KJ/g MS) & $21,0^{\mathrm{b}} \pm 0,06$ & $20,7^{\mathrm{b}} \pm 0,05$ & $21,8^{\mathrm{b}} \pm 0,06$ & $19,5^{\mathrm{a}} \pm 0,02$ \\
\hline Proteína (\% MS) & $45,8^{\mathrm{b}} \pm 0,02$ & $47,9^{\mathrm{b}} \pm 0,16$ & $53,7^{\mathrm{b}} \pm 0,25$ & $36,6^{\mathrm{a}} \pm 0,15$ \\
\hline Lípidos (\% MS) & $13,0^{\mathrm{a}} \pm 0,06$ & $8,3^{\mathrm{d}} \pm 0,18$ & $12,2^{b} \pm 0,03$ & $10,3^{c} \pm 0,04$ \\
\hline Cenizas (\% MS) & $11,3^{\mathrm{b}} \pm 0,02$ & $10,4^{\mathrm{c}} \pm 0,02$ & $9,4^{\mathrm{d}} \pm 0,06$ & $13,7^{\mathrm{a}} \pm 0,05$ \\
\hline Fósforo (\% MS) & $0,8^{\mathrm{b}} \pm 0,05$ & $0,8^{\mathrm{b}} \pm 0,01$ & $0,8^{\mathrm{b}} \pm 0,01$ & $1,3^{\mathrm{a}} \pm 0,02$ \\
\hline $\mathrm{Cr}_{2} \mathrm{O}_{3}(\% \mathrm{MS})$ & $1,54^{\mathrm{a}} \pm 0,10$ & $1,0^{\mathrm{b}} \pm 0,08$ & $1,1^{\mathrm{b}} \pm 0,14$ & $1,06^{\mathrm{b}} \pm 0,08$ \\
\hline \multicolumn{5}{|c|}{ AR: Alimento de referencia a base de pescado Noruego } \\
\hline \multicolumn{5}{|c|}{ T1: Alimento ensayado 1 a base de harina de lombriz (Eisenia andrei) } \\
\hline \multicolumn{5}{|c|}{ T2: Alimento ensayado 2 a base de torta de soja. } \\
\hline \multicolumn{5}{|c|}{ T3: Alimento ensayado 3 a base de afrecho de trigo. } \\
\hline
\end{tabular}


El porcentaje de fósforo del alimento a base de afrecho de trigo (T3) es significativamente superior a los tres alimentos AR, T1, T2, T3. Los valores de proteína estuvieron entre 47,9 \% (T1: harina de lombriz) y 36,6\% (T3: Afrecho de trigo). El contenido de lípidos entre 8,3\% (T1) y 13,0 \% (AR: alimento de referencia). La energía estuvo comprendida entre $19,5 \mathrm{KJ} / \mathrm{g}$ (T3) y 21,8 $\mathrm{KJ} / \mathrm{g}$ (T2: torta de soja). Con respecto al contenido de fósforo en los alimentos este fue de $0,8 \%$ en AR, T1 y T2, y 1,3\% en T3. La concentración de $\mathrm{Cr}_{2} \mathrm{O}_{3}$ en el AR fue significativamente mayor a la presente en los alimentos donde se evaluaba cada materia prima (T1, T2 y T3), lo cual es normal por ocurrir un efecto de dilución, cuando se adiciona el $30 \%$ de la materia prima ensayada.

La abla 3 muestra la composición química de las heces obtenidas por colección durante la experiencia. El ANOVA detectó diferencias significativas $(\mathrm{p}<0,001)$ entre las heces provenientes de las diferentes dietas estudiadas. Se detectaron diferencias significativas entre la materia seca de las heces de las dietas T2 y T4. La proporción en proteína de las heces de la dieta a base de HL es significativamente superior a las tres proporciones en proteínas de las heces de las dietas T2, T3 y T4. Con respecto a la energía del mismo modo se observó que la proporción energética de las heces de la dieta HL presentó la mayor cantidad de energía $(17,0 \mathrm{Kj} / \mathrm{g})$ y la menor proporción de energía la tuvo la dieta del AR $(12,6 \mathrm{Kj} / \mathrm{g})$. 


\section{TABLA 3}

Composición química de las heces colectadas $(\bar{x} \pm D E$.

\begin{tabular}{ccccc}
\hline & \multicolumn{4}{c}{ Heces colectadas por materia prima evaluada } \\
\cline { 2 - 5 } Composición química & AR & T1 & T2 & T3 \\
\cline { 2 - 5 } & $94,3^{\mathrm{b}} \pm 0,16$ & $93,9^{\mathrm{ab}} \pm 0,35$ & $93,3^{\mathrm{a}} \pm 0,12$ & $93,7^{\mathrm{ab}} \pm 0,33$ \\
\hline Materia Seca (\%) & $17,3^{\mathrm{b}} \pm 0,82$ & $23,1^{\mathrm{c}} \pm 0,48$ & $16,9^{\mathrm{b}} \pm 0,30$ & $11,8^{\mathrm{a}} \pm 0,45$ \\
Proteína (\%) & $12,6^{\mathrm{a}} \pm 1,08$ & $17,0^{\mathrm{c}} \pm 0,07$ & $14,1^{\mathrm{ab}} \pm 0,0$ & $14,8^{\mathrm{b}} \pm 1,01$ \\
Energía (KJ/g) & $40,1^{\mathrm{b}} \pm 3,31$ & $29,7^{\mathrm{a}} \pm 1,10$ & $28,2^{\mathrm{a}} \pm 1,27$ & $27,1^{\mathrm{a}} \pm 2,53$ \\
Cenizas (\%) & $2,7^{\mathrm{b}} \pm 0,35$ & $2,1^{\mathrm{a}} \pm 0,06$ & $2,2^{\mathrm{ab}} \pm 0,18$ & $2,1^{\mathrm{a}} \pm 0,04$ \\
Fósforo (\%) & $5,96^{\mathrm{a}} \pm 0,50$ & $4,01^{\mathrm{b}} \pm 0,18$ & $4,11^{\mathrm{b}} \pm 0,09$ & $2.47^{\mathrm{c}} \pm 0.15$ \\
$\mathrm{Cr}_{2} \mathrm{O}_{3}(\% \mathrm{MS})$ & & & & \\
\hline
\end{tabular}

AR: Alimento de Referencia a base de harina de pescado Noruego.

T1: Alimento teste 1 "Harina de lombriz"

T2: Alimento teste 2 "Torta de soja"

T3: Alimento teste 3 "Afrecho de trigo"

Filas com diferente letra difieren significativamente $(p<0,05)$

En relación al contenido de cenizas no hubo diferencias entre las heces de las diferentes dietas a base de HL, TS y AT, pero si con la dieta del AR que presentó el mayor valor (40,1\%). Con relación al contenido en fósforo, todas las heces presentaron valores similares, sin embargo estadísticamente se detectaron diferencias entre las heces de las dietas T1 y T4 en el contendido de este elemento, siendo las heces de la dieta AR la que mostró el mayor valor $(2,7 \%)$. Se detectaron diferencias significativas para la concentración del $\mathrm{Cr}_{2} \mathrm{O}_{3}$ siendo el $\mathrm{AR}$ el que presento el mayor valor $(5,96 \%)$, seguido de las heces de los alimentos $\mathrm{T} 1 \mathrm{y} \mathrm{T} 2$, y el menor valor lo obtuvo la dieta T3. 
Los resultados del CUDa proteica y de energía, proteína digerible y energía digerible, de truchas alimentadas con $30 \%$ de las materias primas harina de lombriz, torta de soja y afrecho de trigo, se muestran en la tabla 4. Debido a que el afrecho de trigo posee un CUDa inferior al $85 \%$, que es lo normal, se decidió no tomarlo en cuenta para hacer comparaciones estadísticas. La harina de lombriz presenta una índice de proteína digerible mayor a la torta de soja $(\mathrm{t}=17,734 ; \mathrm{p}=0,035)$. Para los coeficientes de digestibilidad aparente de proteína, energía y para la energía digerible no se detectaron diferencias significativas entre ambas materias primas.

\section{TABLA 4}

\section{Coeficientes de Digestibilidad (CUDa) de materias primas evaluadas}

\section{Materias primas}

\begin{tabular}{cccc} 
Composición química & "Harina de lombriz" & "Torta de soja" & "Afrecho de trigo" \\
CUDa Proteína (\%) & $90,0^{\mathrm{a}} \pm 1,8$ & $90,1^{\mathrm{a}} \pm 0,1$ & $57,2 \pm 10,6$ \\
\hline CUDa Energía $(\mathrm{KJ} / \mathrm{g})$ & $72,5^{\mathrm{a}} \pm 2,8$ & $75,4^{\mathrm{a}} \pm 1,1$ & $15,2 \pm 17,0$ \\
\hline Proteína digerible $(\%)$ & $63,4^{\mathrm{a}} \pm 1,2$ & $48,3^{\mathrm{b}} \pm 0,1$ & $8,8 \pm 1,6$ \\
\hline Energía digerible $(\mathrm{KJ} / \mathrm{g})$ & $17,1^{\mathrm{a}} \pm 0,7$ & $14,9^{\mathrm{a}} \pm 0,2$ & $2,4 \pm 2,7$
\end{tabular}


La harina de lombriz (E. andrei) presentó un buen coeficiente de digestibilidad proteica del $90 \%$, siendo este valor inferior al valor del $95 \%$ obtenido por Hilton (5) a partir de la lombriz $E$. eugenige, y al valor obtenido por Stafford y Tacon (9) de 94,31\% a partir de un $20 \%$ de harina de lombriz (E. foetida). En comparación con otros autores es superior al obtenido por Pereira y Gomes (12) y Tacon et al, (8) con valores de digestibilidad proteica de 73,4 \% y 83,99 \% a partir de un $25 \%$ y un $34 \%$, respectivamente, de E. foetida incorporada en la dieta con trucha arco iris. La torta de soja también obtuvo un buen valor de CUDa proteica del $90 \%$, mientras que el afrecho de trigo presentó el menor valor (57,2 \%), estos valores están de acuerdo en la literatura, Smith et al. (13) encuentran valores en la trucha situados entre 65 y $89 \%$, las variaciones dependen de la granulometría de la materia prima utilizada. El coeficiente de digestibilidad de energía fue mas alto en la torta de soja $(75,4 \mathrm{KJ} / \mathrm{g})$, seguido por la harina de lombriz con un 72,5 $\mathrm{KJ} / \mathrm{g}$.

La harina de lombriz superó a la torta de soja y afrecho de trigo igualmente en el porcentaje de proteína digerible $(63,4 \%)$ valor que se encuentra por encima del obtenido para la harina de pescado $(59,6 \%)$ reportado Guillaume, et al, (14), y posee una energía digerible $(17,1 \mathrm{KJ} / \mathrm{g})$ similar a la determinada por estos autores en la harina de pescado $(17,3 \mathrm{KJ} / \mathrm{g})$. La TS por su parte tuvo valores de proteína y energía digerible de 48,3\% y 14,9\%, respectivamente, valores que superan los obtenidos por Guillaume, et al, (14) para torta de soja, quienes reportan cantidades de proteína y energía digerible correspondientes de 41,1\% y 13,5\%. El AT por su parte tuvo valores bajos de proteína $(8,8 \%)$ y energía $(2,4 \%)$ digeribles, que contrastan con los obtenidos por Guillaume, et al, (14), que obtuvieron valores de proteína y energía digerible de 15,5\% y $13,9 \%$, respectivamente.

La digestibilidad elevada de la harina de lombriz, se asocia a su composición interesante preliminarmente determinado por diversos autores. De acuerdo a Tacon et al., (8), la lombriz de 
tierra (E. foetida) posee un contenido de proteína cruda entre 50 a $67 \%$ y un buen perfil de aminoácidos esenciales con la posible excepción de Lysina como el primer aminoácido limitante (15) y los aminoácidos sulfurosos (metionina y cisteina). Según ZhenJun (16) esta harina posee un rango de 54,6 a 71,0 \% de proteína en base seca. Sin embargo, Ferruzzi, (17) reporta valores que oscilan entre 65 a $82 \%$.

Los análisis químicos revelan que la lombriz posee $61,8,11,3$ y 8,7 \% de proteínas, grasa y cenizas, respectivamente, y los niveles de metales pesados son bajos y similares al atún de mar (18). La harina de lombriz, además de contener aminoácidos esenciales $(19,20,21)$ y minerales (22) también contiene ácidos grasos esenciales en la nutrición humana.

Análisis de la fracción lipídica de la lombriz de tierra fue una investigación conducida por Hansen y Czøchanska (23) revelando una alta proporción de ácidos grasos insaturados incluyendo ácidos grasos de la serie linolénico $(\omega-3)$ a niveles suficientes para satisfacer los requerimientos de la dieta de trucha arco iris (O. mykiss). Otro aspecto importante de esta harina es su granulometría fina que permite una buena distribución homogénea de sus componentes durante la preparación de las dietas y del proceso digestivo, favoreciendo su CUDa, ventaja suplementaria en la tecnología de alimentos beneficiando su digestibilidad.

La soja, por su parte, es genéticamente mejorada y esto permite eliminar factores antinutricionales, lo cual mejora el CUDa de las dietas donde es incorporada. Las fuentes de proteína de orige n vegetal pueden reemplazar en proporciones variables a la harina de pescado. La utilización de TS en la alimentación de la trucha ha mostrado resultados interesantes, el CUDa de proteína de $96 \%$ de esta materia prima está muy cercana a la del pescado (24). Este valor reportado de CUDa en proteína difiere del obtenido en esta investigación (90,1 \%), sin embargo, su CUDa energética $(75,4 \%)$ es similar al reportado por estos autores $(75 \%)$. Según Cho, et al (24) ciertas materias primas de origen vegetal poseen un bajo contenido en proteínas lo que limita su utilización. Esta particularidad combinada a perfiles en aminoácidos deficientes y 
granulometría variable como es el caso del afrecho de trigo (16\%), determina que esta posea un CUDa deficiente para la alimentación de truchas $<90 \%$, Smith et al, (13).

Agradecimientos: A todo el personal técnico y de investigación de la estación INRA, Saint Pée sur Nivelle, Francia. Al Programa de Cooperación de Posgraduados (PCP) franco-venezolano por el entrenamiento donde se efectuó esta investigación. Al Consejo de Desarrollo Científico Humanístico y Tecnológico (CDCHT) ULA-Mérida, Proyecto FA-342-05-01-ED, por el apoyo otorgado en esta investigación. Al Proyecto FONACIT G-200-5000-869 y a la Oficina de Planificación del Sector Universitario (OPSU) Programa Alma Mater, por la beca otorgada.

\section{CONCLUSIONES}

La harina de lombriz posee una muy buena digestibilidad aparente, en la alimentación de trucha arco iris, semejante a la de la torta de soja.

La digestibilidad proteica y energética fue superior en la harina de lombriz con respecto a las otras dos materias primas evaluadas. Esta puede ser aprovechada para reemplazar a la harina de pescado en ciertas proporciones de formulaciones alimenticias para trucha arco iris.

\section{RESUMEN}

El objetivo principal de esta investigación fue determinar el coeficiente de digestibilidad aparente (CUDa) de la harina de lombriz (HL) y compararla con otras materias primas ya evaluadas, como el afrecho de trigo (AT) y torta de soja (TS) en la alimentación de truchas arco iris (Oncorhynchus mykiss). Esta experiencia se realizó con 15 individuos por tanque en duplicados, con un peso promedio de $100 \mathrm{~g}$, conectados a un sistema automático de recolección de heces. Las harinas de TS y HL presentaron un CUDa proteica de 90,1\% y $90 \%$, respectivamente, al 
compararlas con la del afrecho de trigo (57,2 \%). El CUDa energético fue superior en la TS (75,4 $\mathrm{KJ} / \mathrm{g}$ ) y HL (72,5 KJ/g). Los valores del porcentaje de proteína digerible fueron superiores en la HL (63,4\%); en comparación con la TS $(48,3 \%)$ y AT $(8,8 \%)$. Con respecto a la energía digerible fue del mismo modo superior en la $\mathrm{HL}(17,1 \mathrm{KJ} / \mathrm{g})$, seguido por la $\mathrm{TS}(14,9 \mathrm{KJ} / \mathrm{g})$ y el menor valor para el AT $(2,4 \mathrm{KJ} / \mathrm{g})$. Se concluye que la harina de lombriz posee un valor elevado de digestibilidad proteica y energética.

Palabras claves: Oncorhynchus mykiss; digestibilidad; lombriz; soja

\section{BIBLIOGRAFIA}

1. Zhu S.; S. Chen; R. W. Ardí and F. T. Barrows. Digestibility, growth and excretion response of rainbow trout (Oncorhynchus mykiss Walbaum) to feeds of different particle sizes. Aquaculture Research 2001;32: 885-893.

2. Kaushik S. J. Use of alternative protein source for the intensive rearin of carnivorous fishes. In: Flos, R., Tort, L., Torres L. (Eds), Mediterranean Aquaculture. Ellis Horwood. UK, 1990. pp 125-138.

3. Gomes E. F, Rema, P, Kaushik, S. J. Replacement of fish meal by plant proteins in the diet of rainbow trout (Oncorhynchus mykiss): digestibility and growth performance. Aquaculture 1995;130: 177-186.

4. Tacon A. G.J. Fishmeal replacers: review of antinutrients within oilseeds and pulses. A limiting factor for the aquafeed green revolution? Feeding Tomorrow's Fish Cah Options Mediterr 1997; 22: pp. 153-182.

5. Hilton J. W. Potential of freeze-dried worm meal as a replacement for fish meal in trout diet formulations. Aquaculture 1983; 32: 277-283. 
6. Vanderberg G.W De la Noüe J. Apparent Digestibility comparison in Rainbow trout (Onchorinchus mykiss) assessed Using Three Methods of Feaces Collection and three Digestibility Markers. Aquaculture Nutr 2001 ; 7: 237-245.

7. De la Noüe J. G. Choubert; B. Pagniez; J. M. Blanc et P. Luquet. Digestibilité chez la truite arc-en-ciel (Salmo gairdneri) lors de l'adaptation à un nouveau regime alimentaire. Can J Fish Aquat Sci 1980;37: 2218-2224.

8. Tacon A.G.J., Stafford, E. A. y Edwards, C. A. A preliminary investigation of the nutritive value of three terrestrial lumbricid worms for rainbow trout. Aquaculture. 1983; 35. 187199.

9. Stafford E.A.; Tacon, A.G.J. The nutritional evaluation of dried earthworm meal (Eisenia foetida, Savigny, 1828) included at low levels in production diets for rainbow trout, Salmo gairdneri Richardson. Aquaculture Fisheries Management. 1985; 16: 213-222.

10. Maynard L. A., and Loosli, J. K. Animal Nutrition. $6^{\text {th }}$ Edition, McGraw-Hill, New York. 1969.

11. Sugiura S.H, Dong F.M., Rathbone, C.K., Hardy, R.W. Apparent protein digestibility and mineral availabilities in various feed ingredients for salmonid feeds. Aquaculture 1998; 159: 177-202.

12. Pereira J. and F. Gomes. Growth of rainbow trout fed a diet supplemented with earthworms, after chemical treatment. Aquaculture International 1995; 3:36-42.

13. Smith R, Montle C.P.and Allred, A.C. Effect of leching on apparent digestion coefficients of feedstuffs for salmonids. Prog.Fish-Cult. 1980; 42(4): 195-199.

14. Guillaume J, S. Kaushik, P. Bergot et R. Métailler. Nutrition et alimentation des poissons et crustacés. Institut National de la recherche Agronomique (INRA), Institut Français de Recherche pour 1’Explotation de la Mer (IFREMER), 1999. pp 489. 
15. Tacon A.G.J and Jackson, A.J. Utilisation of conventional and unconventional protein sources in practical fish feeds. In: Nutrition and Feeding in Fish (eds,C.B. Cowey, A.M. Mackie and J.G. Beel). Academic Press: London. 1985; pp 199-145.

16. ZhenJun S. Nutritive value of earthworms. Ecological implications of minilivestock: potential of insects, rodents, frogs and snails: 491-503. College of resources and Environmental sciences, China agricultural University, 100094 Beijing, China. Science Publisher, Inc.; Enfield; USA. ISBN: 1-57808-339-7. 2005.

17. Ferruzzi, C. Manual de Lombricultura. Madrid, Ediciones Mundi Prensa. 1987. pp 137.

18. Medina A. L.; Cova, J. A.; Vielma R. A.; Pujic P.; Carlos M. P.; Torres J. V. Immunological and chemical analysis of proteins from Eisenia foetida earthworm. Food and Agricultural Immunology 15 (3/4) : 255-263. Department of Food Science, School of Pharmacy, University of Los Andes, Mérida, Venezuela. Carfax Publishing, Taylor \& Francis Ltd; Basingstoke; UK ISSN: 0954-0105. 2003.

19. Velásquez L, Herrera C, Ibáñez I. Harina de lombriz. II Parte: Composición de ácidos grasos, factores antinutricionales y tratamiento térmico para control bacterial. Alimentos. 1986; 11 (4): 9-13.

20. Salazar E, Rojas C. Conferencias Curso fundamental de lombricultura. Aspectos GeneralesTeoría. Asociación Colombiana de lombricultores, Asolombriz. Grupo CorpoAndes Mérida. Venezuela. 1992; pp 88.

21. Vielma R, Usubillaga, A, Medina A.L Estudio preliminar de los niveles de ácidos grasos de la harina de lombriz (Eisenia foetida) mediante cromatografía de gases acoplada a espectrometría de masas. Rev Fac Farmacia 2003;45(2):39-44.

22. Vielma R, Carrero P, Rondón C, Medina A. Comparación del contenido de minerales y elementos trazas en la harina de lombriz de tierra (Eisenia foetida) utilizando dos métodos de secado. Saber, Universidad de Oriente, Venezuela. 2007;19(1):25-29. 
23. Hansen, R.P. and Czøchanska, Z. The fatty acid composition of the lipids of earthworms. J Science Food Agricul 1975; 26: 961-971.

24. Cho, C.Y, Slinger S.J, Bayley H.S. Bioenergetics of salmonid fishes: energy intake, expenditure and productivity. Com Biochem Physiol 1982; 73B: 25-41. 